\title{
EDUCACIÓN DE CALIDAD Y CURRÍCULO: UNA MIRADA DESDE LAS TENDENCIAS CONTEMPORÁNEAS Y EL PAPEL DEL DOCENTE
}

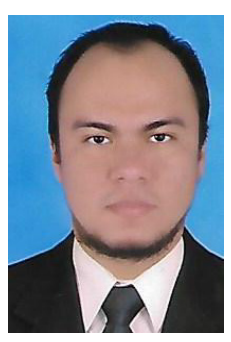

Fecha de recepción: 02/01/2019

\section{Jorge Camilo Portillo Wilches Colombia \\ jportillo_24@hotmail.com}

\section{RESUMEN}

El presente ensayo tiene como objetivo indagar acerca de las características más relevantes de las principales tendencias curriculares contemporáneas, además de dar una mirada al papel que debe desempeñar el docente en cada una de ellas, para que puedan contribuir de una manera efectiva a mejorar la calidad de la educación. Se inicia explicando la complejidad que subyace desde el mismo momento que se intenta definir lo que es la educación de calidad, posteriormente se plantean tres interrogantes: ¿Cuáles son las tendencias curriculares contemporáneas? ¿cuáles son sus características? y ¿qué papel desempeña el docente dentro de ellas? Luego de la revisión documental y en respuesta al primer interrogante se citan entre otras (1) la flexibilidad curricular, (2) el enfoque por competencias, (3) el enfoque por proyectos, (4) la incorporación de manifestaciones del curriculum oculto al curriculum en acción, y (5) la inclusión de las tecnologías de la información y la comunicación (TIC) y a continuación se detallan las definiciones, características y críticas de cada una de ellas. Se destaca que las tendencias estudiadas pueden complementarse, ya que tienen puntos en común como por ejemplo la necesidad de contextualizar y el llamado que se hace a los docentes para que abandonen los paradigmas tradicionales de enseñanza y la zona de confort.

Palabras clave: currículo, calidad, educación, docente

\section{QUALITY EDUCATION AND CURRICULUM:}

A LOOK FROM THE CONTEMPORARY TRENDS AND THE ROLE OF THE TEACHER

\begin{abstract}
The aim of this essay is to investigate the most relevant characteristics of the main contemporary curricular tendencies, as well as to look at the role that the teacher must play
\end{abstract}


in each of them, so that they can contribute in an effective way to improve the quality of Education. It begins explaining the complexity that underlies from the same moment that it is tried to define what is the education of quality, later three questions are posed: What are the contemporary curricular tendencies? what are their characteristics? And what role does the teacher play within them? After the documentary review and in response to the first question are cited among others (1) curricular flexibility, (2) the focus by competencies, (3) the project approach, (4) the incorporation of manifestations of the hidden curriculum to the curriculum in action, and (5) the inclusion of information and communication technologies (ICT) and the definitions, characteristics and criticisms of each of them are detailed below. It is emphasized that the studied trends can be complemented, since they have common points such as the need to contextualize and the call made to teachers to abandon the traditional teaching paradigms and the comfort zone.

Keywords: curriculum, quality, education, teacher

\section{LA EDUCACIÓN DE CALIDAD}

Lograr una educación de calidad no es una tarea sencilla ni siquiera en su definición, y esto se debe a que muchas de las acepciones que se le dan a dicho constructo están directamente relacionadas con la posición de quien la emite, entonces un gobernante podría asociarla con cobertura, un rector con infraestructura, un padre de familia con la intensidad horaria o con la amabilidad que lo atendieron en el colegio, un profesor con la academia o el salario y un niño de escasos recursos con que le den una buena alimentación. Lo interesante del asunto es que, si se hace un análisis a la Norma ISO 9000 de 2015 que trata los sistemas de gestión de la calidad, todos los interesados tienen algo de razón, ya que la responsabilidad en cuestiones de calidad recae sobre los hombros de todas las partes que intervienen, lo que traducido al ámbito educativo significaría que para que haya calidad se deben comprometer desde los vigilantes y aseadores de los establecimientos educativos hasta los ministros y el presidente.

Además, la norma define el término Calidad como el cumplimiento de los requisitos, los cuales no son más que las necesidades y expectativas de las partes interesadas. Lo anterior da luces acerca de la complejidad del tema abordado, por tal motivo resulta sensato tratarlo por partes, decisión que desde el enfoque sistémico tiene mucho fundamento, puesto que la modificación de uno de los componentes debe tener efectos en el resultado del sistema. En este orden de ideas, el presente escrito hace énfasis en algunas de las partes, en especial en el papel que juega el docente, porque tal y como lo manifiestan el informe sobre el desarrollo mundial (2018) y el Informe McKinsey (2007) su rol es protagónico en el aprendizaje de los estudiantes, que en resumidas cuentas es el gran objetivo de los sistemas educativos. 
Como es bien sabido los docentes se enfrentan a grandes retos, el primero es salir de la zona de confort, es decir, dejar de hacer las cosas de la forma tradicional y reconocer los cambios vertiginosos promovidos por la globalización, y el segundo es dejar de culpar a los demás componentes del sistema por los malos resultados en cuestiones de calidad o, en otras palabras, reflexionar para saber cómo se puede aportar al mejoramiento desde la posición en la que se encuentra. Teniendo en cuenta los anteriores retos y tomando como punto de referencia el ciclo PHVA (planear, hacer, verificar y actuar) de la Norma ISO 9000, también llamado el ciclo de la mejora continua, en el que le se le da gran importancia a la planeación para poder lograr el éxito sostenido, se vuelve imperativo que los docentes indaguen sobre aspectos como ¿Cuáles son las tendencias curriculares contemporáneas? ¿cuáles son sus características? y ¿qué papel desempeña el docente dentro de ellas? Ya que los conocimientos adquiridos con las respuestas a dichos interrogantes pueden servir de guía para que se mejoren las prácticas pedagógicas y se elaboren currículos que respondan de una mejor manera a las condiciones del contexto y de los estudiantes.

Luego de una exhaustiva revisión documental hecha para dar respuesta al primer interrogante, se debe mencionar que existe un número significativo de tendencias curriculares contemporáneas, por ejemplo, Barreto (s.f) indica que las principales son:

(1) generación de teoría curricular sustentada en la práctica educativa; (2) construcción de currículos integrados a través de los procesos de cooperación, cogestión, enfoque por competencias y planeación estratégica; (3) flexibilización curricular bajo diferentes modalidades; (4) hibridación o convergencia de especialidades y profundización de las herramientas intelectuales; (5) asunción de visiones inter, multi y transdisciplinares en la construcción curricular; (6) adopción y uso de las Tics en aplicaciones diversas; (7) promoción del desarrollo humano y fortalecimiento de la dimensión ética; (8) incorporación de la pedagogía por proyectos a la elaboración curricular; (9) reformas inclusivas a través de la formación para el compromiso social; (10) incorporación de manifestaciones del curriculum oculto al curriculum en acción; (11) revisión permanente de las ofertas de titulación en las universidades y el perfil de quienes lo forman. (p. 1)

Por su parte Páez (2007) menciona tres tendencias que son la academicista, la humanista y la tecnológica.

También está la visión de Pirela (2007) quien tomando como base la declaración mundial sobre educación superior de 1998 plantea como tendencias:

(1) concebir el currículo como concreción de una teoría pedagógica y como un plan en 
permanente construcción; (2) estructurar los perfiles de ingreso y egreso con base en competencias; (3) garantizar la pertinencia y la calidad en los planes y programas de estudio; (4) asumir nuevos modelos pedagógicos centrados en el aprender a aprender, y en desarrollar los procesos del pensamiento; (5) introducir las tecnologías de información y comunicación como eje de conocimiento, como eje transversal y como nueva forma de conducir los procesos de aprendizaje, y (6) concebir la investigación como eje de conocimiento y como eje transversal, bajo la modalidad transdisciplinaria a modo de vía para abordar la complejidad desde el currículo.(p. 78)

Desde estos autores se puede observar la diversidad de tendencias existentes, sin embargo, también hay coincidencias en sus posturas. A continuación, para dar respuesta al segundo interrogante, se mencionarán las definiciones y características de algunas de dichas tendencias, como son (1) la flexibilidad curricular, (2) enfoque por competencias, (3) enfoque por proyectos, (4) currículo oculto, (5) introducción de las tecnologías de la información y la comunicación. Además, se dará una mirada al papel que debe jugar el docente en cada una de ellas para contribuir verdaderamente a mejorar la calidad de la Educación.

\section{FLEXIBILIDAD CURRICULAR}

En lo referente a la flexibilidad se puede iniciar diciendo que según la RAE algo es flexible cuando es susceptible de cambios, es algo contrario a la rigidez. La flexibilidad curricular Mondragón (2006) la define como la característica o propiedad del diseño que permite y promueve la toma de decisiones de los estudiantes para diseñar el itinerario de su formación profesional en una determinada carrera o programa académico. Para Pedroza (2005) es el proceso de intercomunicación disciplinaria orientado a facilitar la movilidad de los actores académicos, acelerar los flujos de comunicación, conectar el conocimiento con la acción y democratizar la regulación del trabajo académico.

El tema de la flexibilidad curricular se viene mencionando desde hace mucho tiempo, en la actualidad muchas universidades la destacan como uno de sus pilares, pero la realidad demuestra que no es un proceso sencillo, ya que contrario a lo que muchos creen, requiere estructura, control y vas más allá de simples enunciados en la planeación. Cuando hay una verdadera flexibilidad curricular los estudiantes tienen un papel protagónico, en el que deben ser responsables de muchas decisiones referentes a su formación, pero para que esto suceda se deben dejar atrás algunos paradigmas que no consideran a los estudiantes como seres capaces de pensar, reflexionar o interpretar.

Para entender los ámbitos en que se puede aplicar, Díaz (2005) menciona que la flexibilidad 
curricular puede plantearse por lo menos de dos formas: la primera referida a la apertura de límites y de las relaciones entre campos, áreas y contenidos del currículo; y la segunda, al grado de apertura de los cursos y las actividades académicas de acuerdo con las necesidades de los estudiantes. Teniendo en cuenta lo anterior Pedroza (citado por Escalona, 2007) afirma que las características de un currículo flexible son:

a) Adecuar permanentemente los nuevos conocimientos a los procesos de formación, lo que implica la actualización permanente dela disciplina;

b) Promover la capacidad de decisión en el alumno en el momento de elegir las asignaturas o módulos que debe cursar y lo ayuda a determinar el ritmo de sus estudios;

c) Fomentar el trabajo colegiado de docentes e investigadores al darle una nueva función a la academia;

d) Propiciar la comunicación horizontal y vertical de los contenidos al evitar la rigidez de materias secuenciales, con lo que se mejora la comunicación de todo el plan;

e) Facilitar la movilidad de los actores académicos, tanto estudiantes como docentes, primero hacia el interior de la institución, pero también se puede lograr esto con otras instituciones y países;

f) Optar por un aprendizaje integral mediante el contacto del estudiante con el mundo circundante ya que al tener acceso a una amplia gama de áreas disciplinares, se lo forma en diversas estrategias que le permiten ampliar sus expectativas de aprendizaje;

g) Vincular la Universidad con otras instituciones de la sociedad;

h) Buscar la formación de un hombre comprometido con sus circunstancias, reflexivo y polivalente (conocimiento y experiencia panorámica en el campo laboral de su disciplina);

i)Estimular la interdisciplinariedad, disciplinariedad y transdisciplinariedad al situar la disciplina en el área del conocimiento y al actuar en la resolución de problemas reales;

j)Redimensionar el papel que juega el docente como sujeto social que convoca al alumno a establecer el diálogo y a explorar sus propias intencionalidades, y

k) Hacer viable el sistema de créditos, aspecto que estudiante representa un serio obstáculo porque cada institución evalúa con diferentes parámetros.

Todas estas características del currículo flexible pueden contribuir a que se mejore la calidad de la educación en cuanto busca formar profesionales capaces de tomar decisiones responsables y que resuelvan problemas del contexto. Así mismo le da un nuevo significado a la labor docente.

\section{Enfoque por competencias}

Para entender el enfoque de competencias en el currículo se hace necesario en primera instancia definir lo que es una competencia. Para el Icfes (1999) es un conjunto de acciones que 
el sujeto realiza cuando interactúa significativamente en un contexto determinado, definición que se resume en: un saber hacer en contexto. La competencia también puede ser entendida como una “actuación idónea que emerge de una tarea concreta, en un contexto con sentido” (Bogoya, 2000). Una definición más extensa la brinda López (2011) quien plantea que la competencia es un sistema de conocimientos, habilidades y actitudes que son utilizados de modo interactivo en la ejecución oportuna de tareas y en la resolución de problemas más o menos complejos en el marco de una determinada situación y movilizando los recursos y medios disponibles en el ambiente específico en que se opera, teniendo en cuenta que este contexto puede ser tanto académico como laboral.

Como se puede evidenciar el término competencia tiene múltiples definiciones, de las cuales se pueden rescatar algunos elementos comunes como son el conocimiento, la acción y el contexto.

Las principales razones para tener un currículo con enfoque en competencias según López (2011) son conseguir la coherencia e integración de todo el sistema educativo, reorientar la misión de la educación superior en el siglo XXI, conectar con el inestable mundo laboral, abrir una puerta a la interdisciplinariedad, renovar las metodologías docentes, mejorar la evaluación del aprendizaje, evaluar la práctica docente y posibilitar la convergencia. En esta justificación se destaca el nuevo rol que tiene el docente, ya que debe romper el paradigma de la educación tradicional, en el que principalmente se desarrollan clases magistrales a una audiencia que permanece en silencio. Además, se debe trabajar en la solución de problemas en múltiples contextos, buscar la coherencia con las demandas del mundo laboral, realizar evaluaciones formativas y ver la evaluación de su propia práctica como una oportunidad de mejora. Diseñar un currículo por competencias implica construirlo sobre la integración de varias disciplinas, así como tener en cuenta los procesos y no solo los contenidos.

En el plano de las razones para implementar el enfoque por competencias Tobón (2007) plantea que es necesario para aumentar la pertinencia de los programas educativos, gestionar la calidad y responder a la política educativa internacional. La pertinencia se refiere a que la educación sirva para enfrentar los retos y problemas que aparecen en el contexto, la gestión de la calidad ocurre gracias a la evaluación de docentes y estudiantes, y responder a la política internacional significa ir en la misma vía de las nuevas tendencias curriculares.

Al respecto Gonzalez (2007) plantea que en la formación de profesionales es necesario realizar cambios metodológicos, didácticos y actitudinales que promuevan la participación, cooperación y estimulen el pensar del alumno, en la medida que se construyen los conocimientos junto al docente, apostando por un estudiante que aprenda a aprender, con una actitud crítica y capacidad 
de responder y actuar ante el cambio. En resumen, manifiesta que un enfoque por competencias en el currículo requiere los siguientes cambios:

- Centrar la formación en el aprendizaje y no en la enseñanza y reconocer los aprendizajes previos.

- Un pilar clave disponible por la organización educativa es el docente. La formación de docentes en los principios, implicaciones y herramientas del enfoque de competencia es fundamental e indispensable en el proceso de cambio.

- Una mayor relación entre la teoría y la práctica, que le permita a los alumnos contextualizar los conocimientos adquiridos.

- Lo anterior obliga a desarrollar y mantener una mayor vinculación con el sector productivo y a incorporarlo en los procesos de planificación curricular.

- Los procesos de evaluación deben ser completamente reformulados, como modo de garantizar el dominio de los aprendizajes.

Hasta este punto se han tratado las bondades del enfoque, sin embargo, el currículo con enfoque por competencias también es objeto de algunas críticas, entre las que se destacan las hechas por Andrade (2008) en cuanto a la falta de profundización en el aspecto metodológico, es decir, en el cómo, que es claramente el que conduce a la acción. Otra dificultad tiene que ver con el proceso de formación de los docentes, lo cual requiere mucho compromiso y tiempo. En este sentido Moreno (2018) expresa que existe el riesgo inminente que el profeso $\neg$ rado caiga en la simulación y siga haciendo las cosas igual que antes. Muchos profesores abordan las reformas con una postura escéptica pensando que eso traerá una reducción del nivel, que sa $\neg$ crifican el conocimiento, que vuelven la espalda a la cultura o que son invenciones de algunos tecnócratas tendenciosos. Estas representaciones sirven como razones válidas para no actuar.

También sobresale la crítica de Díaz Barriga (citado por Moreno, 2008) cuando manifiesta que en general existe prisa por aplicar una nueva tendencia o una nueva estrategia sin una reflexión conceptual, como si existiera presión por la realidad educativa para llevar a cabo acciones. Este tema tiene relación con la necesidad del sistema de realizar innovaciones, pero la dificultad principal es que muchas veces estas acciones no se realizan de una forma sólida dado que la innovación es más una declaración verbal que una acción realizada por parte de los docentes a partir de un convencimiento de la importancia de la innovación o por lo menos de un dominio conceptual y técnico del significado de la misma.

En definitiva, los distintos autores coinciden en un punto y es señalar que cualquier propuesta de reforma curricular, al final recae sobre los hombros del profesor, quien, mediante sus prácticas 
en el aula puede promo $\neg$ ver la reforma o traicionarla. Queda claro de esta manera que un currículo por competencias puede llegar a ser una excelente opción de formación para los profesionales, solo cuando hay una adecuada articulación y comprensión por parte de quienes lo ejecutan.

\section{Enfoque por proyectos}

En la norma ISO 9000 de 2015 se entiende por proyecto al "proceso único, consistente en un conjunto de actividades coordinadas y controladas con fechas de inicio y de finalización, llevadas a cabo para lograr un objetivo" (p.20). Por su parte Tobón (2006) lo define como "un conjunto de actividades sistemáticas y elaboradas que se ejecutan con el fin de resolver un determinado problema" (p. 1).

Plantea Tobón (2006) que el trabajo por proyectos dentro del currículo consiste en:

La construcción con los estudiantes de un problema, el diseño de estrategias de resolución, su ejecución y valoración, buscando el trabajo en equipo y la participación de otras personas (pueden ser pares, familia, docentes, empresas, institucionales no gubernamentales, grupos informales y otros miembros de la comunidad), teniendo como base la formación y/o consolidación de un determinado conjunto de competencias definidas dentro del Proyecto Educativo Institucional. (p. 1)

Los currículos se pueden estructurar por proyectos como propuesta alternativa al trabajo por contenidos, que como ya se ha mencionado, se caracteriza por su rigidez. El trabajo por proyectos busca la solución de problemas del contexto, siendo este un punto común con el enfoque por competencias. Además, desde un proyecto se puede promover el desarrollo de competencias básicas, genéricas y específicas. Teniendo en cuenta lo anterior se puede afirmar que este enfoque va en la misma vía que el de competencias, por tal motivo podrían ser complementarios y aportar al mejoramiento de la calidad de la educación.

La importancia de la metodología por proyectos en educación según Tobón (2006) radica en que en ella se integran aspectos fundamentales en la formación, tales como: observación de fenómenos naturales y/o sociales, análisis de documentos, consulta bibliográfica, interpretación de situaciones, construcción de instrumentos de conocimiento, desarrollo de la creatividad, afianzamiento de las competencias comunicativas y trabajo en equipo.

El trabajo por proyectos enmarcado dentro del currículo puede aportar a la construcción de aprendizajes significativos, ya que los estudiantes se deben enfrentar a problemas del contexto que tienen sentido para ellos. Esto a la vez hace que haya motivación, trabajo colaborativo y se generen 
ideas.

\section{Currículo oculto}

Con respecto al origen del concepto se debe decir que este fue acuñado por Jackson (1960) (citado por Barreto, s.f) quien señala que "la multitud, el elogio y el poder que se combinan para dar un sabor específico a la vida en el aula forman colectivamente un curriculum oculto que cada alumno (y cada profesor) debe dominar para desenvolverse satisfactoriamente en la escuela" (p. 15).

En la actualidad la mayoría de los docentes son conscientes de que deben incluir algunos cambios en sus prácticas pedagógicas, sin embargo, se cohíben porque tienen que desarrollar los programas de curso que están en el currículo formal, con el objetivo de que los estudiantes obtengan resultados favorables en las evaluaciones externas. Se convierte entonces el currículo oculto como lo manifiesta Santamaría (2013) en la oportunidad para que el docente proyecte su práctica hacia el desarrollo de otros aprendizajes por parte del estudiante, que no están explícitos en ningún currículo formal.

Se puede agregar que "el currículo oculto representa un trasfondo de normas, valores y reglas latentes en el proceso formativo, que los estudiantes han de asumir y adoptar para desempeñar convincentemente un papel social" (Wren 1999, citado por Rodríguez 2012). Esto quiere decir básicamente que mucho de lo que se enseña y se aprende no ocurre dentro de la formalidad del aula de clases. Según Bennet (citado por Rodríguez, 2012) Desde un punto de vista práctico, se podría definir como el conjunto de reglas, regulaciones y rutinas institucionales no escritas. Para Díaz (2005) el currículo oculto es un buen objeto de estudio, porque permite dar cuenta de esos procesos de socialización que se llevaron a cabo en la realización de la acción escolar. Da cuenta, precisamente, de aquellos que no son intencionados, aunque su efectividad no se puede negar.

De las anteriores posturas se extraen dos aspectos de relevancia, el primero es la responsabilidad que tiene el docente en lo relacionado con la apropiación intencionada de los elementos que conforman el currículo oculto, tanto para entender la realidad educativa como para mejorarla; el segundo es la necesidad de que haya una alineación entre el currículo formal y el currículo oculto para poder potencializar los aprendizajes deseados.

\section{Introducción de las TIC en el currículo}

En la actualidad las instituciones educativas atienden jóvenes que nacieron con las nuevas tecnologías, son los llamados nativos digitales. Ellos toda la vida han tenido acceso a computadores, teléfonos inteligentes, cámaras, internet y juegos de video. Por ese motivo y para incrementar la motivación de los alumnos estos aspectos se deben tener en cuenta en el diseño de los nuevos 
currículos (Marchesi, 2009).

Tradicionalmente los procesos de enseñanza se han organizado en torno al libro y al profesor como transmisor de conocimientos, sin embargo, con la aparición de las TIC aparecen nuevos caminos para obtener la información y el docente está obligado a replantear su papel y sus prácticas. Este es un enfoque que no entra en contradicción con el enfoque por competencias ni con la flexibilidad curricular. En este sentido, según Padilha (citado por Iriondo, 2013) el debate sobre TIC y currículo incorpora las competencias de selección y organización de información, de comunicación, de trabajo en red, o colaboración, así como las operaciones mentales, habilidades y actitudes implícitas en esas tareas.

En lo referente a las ventajas de integrar las TIC en el currículo autores como Arévalo y Gamboa (2015) reconocen que el uso de las TIC puede mejorar la calidad de la edu $\neg$ cación, innovar en las prácticas educativas, enriquecer los aprendizajes, desarrollar competencias personales, profesionales y también se espera que su inclusión en la escuela reduzca la brecha digital, la pobreza, y contribuya con el desarrollo social y económico de los países.

La integración de las TIC no es un proceso aislado, ya que debe haber un reconocimiento de las políticas, proyectos educativos y del contexto en general. Las políticas se convierten en el marco que puede potencializar el proceso y la contextualización hace que se puedan desarrollar prácticas pedagógicas que estén alineadas con las necesidades institucionales y la realidad social.

A pesar de las múltiples ventajas que conlleva el uso pedagógico de las TIC, en países como Colombia hace falta un largo camino por recorrer, la experiencia muestra que se han presentado algunas iniciativas en cuestiones de cobertura, sin embargo en la zona rural por ejemplo persisten las dificultades en cuanto al acceso, al respecto Arévalo y Gamboa (2015) afirman que esta incorporación se encuentra en un nivel básico dedicado en un alto porcentaje a la dotación de infraestructura y a los procesos de cobertura, sin avanzar significativamente en la incorporación pedagógica y didáctica y en el mejoramiento de los procesos de cali $\neg$ dad educativa dados estos recursos tecnológicos. En resumidas cuentas, aún se presenta la brecha digital, porque además de tener inconvenientes de cobertura, encontramos una gran cantidad de maestros tratando de aprender sobre las tecnologías, mientras que en otros países ya los docentes están mediando sus prácticas con las TIC de una manera fundamentada e institucionalizada.

Con respecto a las posibilidades de integración curricular de las TIC Gutiérrez (2007) plantea tres opciones: 
- Incluir sus contenidos en distintas áreas

- Crear asignaturas específicas para el estudio de los medios, las que actualmente suelen ser optativas

- Considerar la educación para los medios como contenido transversal al currículo y una responsabilidad de todos.

Desde la calidad total obviamente el compromiso con la Integración debe ser de todos, desde los directivos y administrativos hasta los docentes y estudiantes. Sin embargo, se resalta en esta parte el papel del docente nuevamente, ya que requiere un notable compromiso y tiene la necesidad de capacitación para que no se convierta en un obstáculo.

Con respecto a la formación de docentes Gutiérrez (2007) advierte sobre algunos peligros. Manifiesta que, aunque el manejo de los nuevos equipos es necesario no se debe priorizar esto. El énfasis debería estar más bien en la reflexión sobre la presencia de las TIC en la sociedad, sobre la influencia en la vida de las personas, sus ventajas e inconvenientes. Así las destrezas en el manejo de equipos y programas vendrán por añadidura.

Además, Gutiérrez (1997) señala tres importantes dimensiones de la formación del profesorado en TIC: conocimientos y competencias sobre las posibilidades de las TIC como recurso didáctico, potencial educativo, conocimiento del currículo oculto, las implicaciones y consecuencias de las TIC en los aprendizajes formal e informal y conocimiento de los contextos.

De esta manera se ratifica que el docente juega un papel fundamental dentro de la integración de las TIC en el currículo, y que a pesar de necesitar de las políticas de gobierno para que se logren los objetivos de calidad, desde su posición puede aportar grandemente si vas más allá de la mera apropiación instrumental, para llegar a una reflexión que le permita realizar una práctica pedagógica fundamentada.

\section{Apuntes finales}

Por cuestiones de extensión del documento no se entró en detalle en todas las tendencias curriculares contemporáneas citadas al inicio, pero con el hecho de mencionarlas queda la inquietud para que los docentes indaguen más acerca de ellas.

Del análisis de las diferentes tendencias en conjunto se puede concluir que no son excluyentes, es decir, el hecho de aplicar una no implica que no se puedan tomar principios de otra. Como se evidenció el enfoque por competencias, la flexibilidad, el currículo oculto, la utilización de las TIC 
y los proyectos, se deben considerar como enfoques que se complementan y que apuntan hacia la mejora de la calidad de los sistemas educativos.

En todas las tendencias estudiadas el docente juega un papel fundamental y recibe un llamado a dejar de actuar bajo el paradigma de la educación tradicional. Esto quiere decir que, aunque no es un proceso sencillo los docentes deben salir de la zona de confort para poder afrontar los nuevos retos que trae una realidad en constante cambio.

En conclusión, son muchos los aspectos que se deben tener en cuenta para que haya una educación de calidad, sin embargo, dicha situación no debe ser la excusa para que ninguna de las partes actué, sino por el contrario, debe ser un aliciente para que cada uno de los involucrados en el proceso cumpla su papel de una forma satisfactoria. En particular los docentes se deben comprometer con la ejecución y su propia formación que son los dos aspectos donde recaen las principales críticas.

\section{BIBLIOGRAFÍA}

Andrade, R. (2008). El enfoque por competencias en educación. Ideas Conciteg. Año 3, Núm. 39

Arévalo, M. y Gamboa, A. (2015). TIC en el currículo de matemáticas. Una orientación desde el marco de las políticas y proyectos educativos. RIIEP, Vol. 8, N. . 1. Pp 169-187.

Bogoya, D. (2000). Competencias y Proyectos Pedagógicos. Capítulo: Educar para el desarrollo de las competencias: Una propuesta para reflexionar. Santa Fe de Bogotá. Universidad Nacional de Colombia.

Díaz, A. (2005). La educación en valores: Avatares del currículum formal, oculto ylos temas transversales Universidad Nacional Autónoma de México Centro de Estudios sobre la Universidad.

Díaz, M. (2005). "Flexibilidad y organización de la educación superior”, 63-117, en Flexibilidad académica y curricular en las instituciones de educación superior, René Pedroza Flores, Bernardino García Briceño, comps. México: M.A. Porrúa.

Escalona, L. (2007) Flexibilidad curricular: elemento clave para mejorar la educación bibliotecológica. Centro Universitario de Investigaciones Bibliotecológicas de la UNAM. Investigación bibliotecológica, Vol. 22, Núm. 44, enero/abril, 2008, México, ISSN: 0187358X. pp. 143-160

González, E. (2007). Formación universitaria por competencias. Centro Interuniversitario de Desarrollo CINDA. Recuperado de https:/www.researchgate.net/publication/275275474 
Gutiérrez, A. (1997). Educación multimedia y nuevas tecnologías. Madrid: Ediciones de la Torre.

Gutiérrez, A. (2007). Integración curricular de las TIC y educación para los medios en la sociedad del conocimiento. Revista Iberoamericana de Educación, No. 45. Madrid, España, pp 141-156.

ICFES (1999). Nuevo examen de estado, Propuesta General. Santa Fe de Bogotá:

Icontec (2015). Norma ISO 9000 de 2015. Vocabulario del sistema de gestión Calidad.

Informe sobre el desarrollo mundial (2018): Aprender para hacer realidad la promesa de la Educación.

Iriondo, W. (2013). El currículo y la educación a distancia. RIED v. 16: 1, pp 109-132

López I. (2011). Un giro copernicano en la enseñanza universitaria: formación por competencias. Universidad de Sevilla. Facultad de Ciencias de la Educación. Departamento de Didáctica y Organización Educativa. Sevilla, España. Revista de Educación, 356. Septiembre-diciembre 2011, pp. 279-301

McKinsey (2007). Cómo hicieron los sistemas educativos con mejor desempeño del mundo para alcanzar su objetivo

Mondragón, H. (2006). Glosario con terminología básica de apoyo al diseño y ejecución curricular 1, comp., Colombia: Universidad Javeriana de Cali, Colombia. en http://portales. puj.edu.co/didactica/Archivos/Glosario/GLOSARIO.pdf consultado el 30 de Marzo de 2019. Moreno, T. (2010). El currículo por competencias en la universidad: más ruido que nueces. Revista de la Educación Superior Vol. XXXIX (2), No. 154, pp. 77-90. ISSN: 0185-2760

Páez, V. (2007). Formación profesional pedagógica, tendencias curriculares y contexto educativo APROXIMACIONES DESDE LA REALIDAD CUBANA. Revista Varona. núm. 45, julio-diciembre, pp. 9-15 Universidad Pedagógica Enrique José Varona La Habana, Cuba. Pedroza, R. (2005) "La flexibilidad académica en la universidad pública", pp. 19-41, en Flexibilidad académica y curricular en las instituciones de educación superior, comps. México : M.A., Porrúa.

Pirela, J. (2007). Las tendencias educativas del siglo XXI y el currículo de las escuelas de Bibliotecología, Archivología y Ciencia de la Información de México y Venezuela.

Rodríguez, F. (2012) Proceso de Bolonia: el currículo oculto Facultad de Ciencias de la Salud. Universidad de Las Palmas de Gran Canaria. España.

Santamaría, B. (2013). Pensamiento visible y currículo oculto. Revista Electrónica de Psicología Social «Poiésis»

Tobón, S. (2006). Método de trabajo por proyectos. Madrid: Uninet.

Tobón, S. (2007). El enfoque complejo de las competencias y el diseño curricular por ciclos propedéutico. Grupo CIFE (www.cife.ws) 